\title{
LOS PROTOCOLOS DEL ARCHIVO MUNICIPAL DE ELCHE: APLICACIONES Y POSIBILIDADES DE TRABAJO*
}

\author{
Pedro Félix JOVER IBARRA \\ Rafael PLA GRAU \\ Universidad de Alicante
}

* Este trabajo reúne las Memorias de Licenciatura que bajo la dirección de Enrique Giménez López fueron defendidas en noviembre de 1982 y enero de 1983 en la Facultad de Filosofia y Letras de la Universidad de Alicante.

Los protocolos notariales han sido una de las fuentes a las que tradicionalmente ha recurrido el investigador de la historia social. Su utilización, quizá como consecuencia de este hecho, no ha sido siempre la misma. En la actualidad, superando cualquier consideración basada en el interés por el dato individual y aislado, todo estudio apoyado en este tipo de documentación se basa en su tratamiento cuantitativo, en su valoración como fuente cuantificable, susceptible de ser utilizada de forma serial(1).

Este ha sido el principio desarrollado por los autores de la escuela de los "Annales"; primero en las grandes tesis de historia rural, en las que la información proporcionada por las series de protocolos se conjunta con la obtenida de otros documentos de diverso origen: parroquiales, fiscales y señoriales, por ejemplo. $Y$ en segundo lugar, en los trabajos de autores como M. Vovelle, P. Chaunu, F. Furet o A. Daumard, realizados sobre las 
conclusiones extraidas fundamentalmente del análisis de un único tipo de acta, como ocurre con los testimonios en los estudios de M. Vovelle y P. Chaunu, y con las cartas nupciales en los de A. Daumard y F. Furet.

En España estos planteamientos han tenido su eco en el núcleo vinculado a la Universidad de Santiago de Compostela, dirigido por el profesor A. Eiras Roel (2), no olvidando las aportaciones de las Universidades de Sevilla y La Laguna.

En este trabajo, partiendo de las posiciones metodológicas que hemos señalado, intentaremos poner de relieve algunas de las posibilidades y problemas planteados en el estudio de los protocolos notariales conservados en el Archivo Municipal de Elche (A. M. E.).

De entrada, hemos de dejar claro que se trata de una evaluación realizada sobre la información que revelan las minutas de los oficios de don Pedro Pasqual de Ganga(3), escribano real y notario público de los del Número del Reino de Valencia que ejerce su actividad en Elche, y de Joseph Ardid (4), escribano real de Crevillente.

Teniendo en cuenta estas limitaciones, expondremos algunas de las aplicaciones de este tipo de fuente en los tres aspectos básicos en los que la historiografía actual las ha utilizado con mayor frecuencia: economía, organización social y cultura(5).

\section{Economia}

Dentro de este gran campo trataremos sus tres actividades fundamentales: sector agropecuario, industria y comercio, esferas económicas que, en ocasiones, es difícil separarlas totalmente ya que se complemen$\tan$.

1.-Sector agropecuario: En un medio eminentemente agrícola, como el que estudiamos, es lógico que las escrituras que reflejen la vida y actividades agrarias sean numerosas, siendo su peso específico muy importante dentro de la documentación. Asi, este sector queda reflejado en los arrendamientos, las ventas, las obligaciones y las cartas de pago, y de forma indirecta en los inventarios, cartas nupciales y testamentos.

El área rural que se señala en ellas se reduce a los términos municipales de los lugares en que trabaja cada escribano. En el caso de Elche con mayor frecuencia se citan las partidas de Valverde, Jubalcoy, Beniay, la Torre del Llano, Altabix, La Baya...; y en el de Crevillente las del Derramador, Deula, Cachapet, Carga y el Campillo. Sólo, a veces, se hace referencia a centros urbanos próximos: Aspe, Catral, Alicante, San Phelipe, etc.

Gracias a que, en la mayor parte, se detallan las características de la tierra arrendada, vendida o poseída, podemos llegar a conocer los cultivos 
que se practicaban en la zona. Cultivos típicos como las viñas, olivos, algarrobos, almendros, higueras, palmeras, barrilla y cereales, cebada y trigo fundamentalmente(6). Hay que señalar que el estudio de los diferentes tipos de cultivo a través de las escrituras notariales en un período secular, puede mostrarnos cómo aumentan y disminuyen en su producción diferentes cultivos y cómo unos desplazan a otros.

Teniendo en cuenta todos estos datos, se confeccionaría un mapa comarcal donde figurasen las distintas partidas y los cultivos que en cada una de ellas predominasen, delineando así un mapa geográfico-agricola para un determinado período(7).

Más dificil es conocer a través de los protocolos el tipo y periodicidad de las labores agrícolas realizadas. La única fuente apta para ello son los arrendamientos, que en sus condiciones concretan algunas de las ocupaciones de los arrendatarios. De forma más general se alude a la escarmonda en los olivares (8) y al estercolado en las tierras de labor (9). En menor medida, se mencionan labores de carácter estacional, como el realizar dos cultivos a la viña, uuno en invierno labrándola a dos rejas y cavando las cepas y altaras, y el otro en verano cavándola a legón„ (10); la escarda y siega de las mieses(11).

Relacionado con estas tareas es imprescindible hablar del instrumental agrícola. Con las informaciones obtenidas de cartas nupciales e inventarios es posible trazar la evolución de los útiles de trabajo en un período de tiempo amplio. Según nuestras referencias, se adivina el mantenimiento de un utillaje tradicional, del que no obstante conocemos muy poco sus características. Normalmente se citan los instrumentos sin más: arados, legones, corbillas, hoces, ganchos de sacar estiércol, azadones... Sólo en ocasiones se describe con más detalle la herramienta, "un arado con orejeras de hierro y con una reja nueva con un peso de seis libras" (12).

Por otra parte, concretar a través de los protocolos, tal y como nos aparecen, la estructura de la propiedad ofrece algunos problemas. Inicialmente se nos plantea la necesidad de conocer con la mayor aproximación posible el lugar ocupado por los arrendamientos dentro de las relaciones contractuales que tienen lugar en el ámbito rural, es decir, habria que ver qué espectro de la población se contiene en ellos y tener en cuenta las explotaciones reguladas por otro tipo de contratos, como la enfiteusis. Sólo considerando estas particularidades se podría conocer con exactitud la extensión media de las unidades de explotación y quiénes son los propietarios. No obstante, todos estos datos deben ser comparados con los padrones y los registros, como los cabreves, mucho más adecuados para el estudio de la propiedad y de sus variaciones.

Teniendo presentes estas dificultades hemos obtenido alguna conclusión al respecto. En primer lugar, en el caso de Elche tenemos que 
dos tercios de los arrendadores pertenecen al estamento nobiliario, pudiendo citar como representativos a don Benito Ruiz de Musoles, don Pedro Miralles de Ymperial y, sobre todo, los miembros de la familia Perpiñán(13). Estos nobles están casi siempre representados por sus apoderados o administradores, destacando el doctor don Gaspar Esclapez(14), Josef Llofrui de Coquillat(15), Ygnacio Ruis de Lope y Bru(16) y Geronimo Molla de Torres(17). En las escrituras de ventas actuarán como compradores, apropiándose principalmente de las extensiones más grandes. Es de destacar como acaparadores de tierras por sus compras en un corto periodo de tiempo a don Pedro Leguey, administrador de la excelentísima señora Condesa Duquesa de Benavent y Arcos; don Antonio de la Murere, capitán retirado agregado a la plaza de la ciudad de Alicante, y las hermanas Perpiñán y Tárrega, que efectúan gran número de compras, sobre todo, en el año 1798, arrendando, en muchas ocasiones, las tierras a sus antiguos propietarios. En resumen, a juzgar por los arrendamientos que ofrecen y por las compras realizadas, esta pequeña nobleza goza, en su mayoría, de una gran extensión de tierras de su propiedad.

Aparte de ello, nos encontramos con individuos de un nivel medio de vida, es decir, con comerciantes que han invertido en tierras y con algunos campesinos bienestantes, como es el caso de Gaspar Meléndez de Miralles. Estos disfrutan de una mediana o, sobre todo, pequeña propiedad rústica.

En general, en cuanto a la extensión de las tierras arrendadas predominan las pequeñas y medianas parcelas, no sobrepasando la mayoria de 50 tahúllas (th.); algunas llegan hasta 100 th., sólo tres pasan de 200 th. y, excepcionalmente, se llega a 632 th. (18) y 730 th. (19) arrendadas. Por otro lado, la propiedad vendida es mayoritariamente pequeña - abundan las ventas de tierra de $2,3,4,5,6,7$ y 8 th. - y mediana - de 30 a 40 th. - . Las propiedades más extensas que se vendieron fueron de 63 th. y 86 th., es decir, las propiedades rústicas que se venden son de pequeño tamaño. Hay que señalar que a veces indican tan sólo que se trata de un bancal o un trozo de tierra.

Por el contrario, en el caso de Crevillente la información ofrecida por los protocolos de J. Ardid no es tan abundante, de ahí que no se pueda concretar la situación social de los propietarios de tierras según las escrituras. Los nombres que con más frecuencia se repiten son el de Jaime Mas de Ardid y el de Cayetano Mas. Entre los arrendadores aparecen algunos notarios y abogados, siendo el resto, pues, de pequeños comerciantes y campesinos. La superficie arrendada es muy variable y oscila entre el máximo de 198 th. (20) y el minimo de 8 th.(21). Igualmente hay que señalar que se tropieza con el problema de que en el $50 \%$ de los casos no se expresan las dimensiones concretas de las tierras. 
Por otro lado, este tipo de escrituras nos permite conocer uno de los sistemas más importantes de explotación agraria: los arrendamientos a corto plazo, sobre todo de seis años de duración, que coexistiendo perfectamente con la enfiteusis se convertirán en la relación agraria dominante. Este triunfo de las relaciones contractuales en el mundo rural será llevado a cabo por la pequeña nobleza y por los comerciantes o campesinos enriquecidos (22). Este sistema establecerá unos pagos en metálico anuales para cuyo efectivo se contará con la ventaja de los plazos. No obstante, todavía subsisten otros pagos en especie de carácter simbólico semejantes a las "aldehalas", cuya significación económica es mínima, dirigida al aprovisionamiento del propietario, como son la entrega de gallinas y pollos por Navidad o San Juan, aspecto éste de cierto resabio señorial(23).

Sin salirnos de esta temática, a través de las ventas, escrituras en las que se registran las variaciones de la propiedad, será posible trazar una evolución general tal y como expresa J. Jacquart(24): qué se vende, en qué cantidad, a qué ritmo, quién compra y a qué precio. Con ello está a nuestro alcance el estudio del movimiento del valor de la propiedad y de las vinculaciones de los distintos grupos sociales con la tierra. Pero las dificultades que aparecen son muchas. Como ya hemos dicho, en bastantes ocasiones no se concreta la superficie vendida. Al margen de ello influyen otros factores en la determinación de los precios: el momento en que se realiza la venta, antes o después de la cosecha, el estado de los plantados, la proximidad con las tierras del comprador, el establecimiento de los plazos, etc.

Una segunda serie numérica que sería factible obtener, esta vez a partir de los arrendamientos, es el movimiento de la renta inmobiliaria, que permite conocer quiénes son los beneficiarios de la evolución de la coyuntura y en qué medida incrementan sus ingresos. En resumen, contando con lo ya dicho, podemos indicar que a partir de los precios de las propiedades de regadio o de secano se podrian formar curvas que reflejen la evolución del precio de los arrendamientos, incluso estimando esta evolución en función de los distintos cultivos, pudiendo constatar sus momentos alcistas y sus depresiones. Igualmente, teniendo una información serial, por unidad de superficie y en función de las características y situación de las propiedades se puede llegar a una evolución general de los precios de las tierras.

Dentro de este primer sector económico, el segundo aspecto es el de la ganadería. Las noticias sobre la clase de ganado se pueden recoger a través de la información contenida en los inventarios, cartas nupciales, y en las obligaciones y cartas de pago que hacen referencia a la compraventa de animales relacionados con la vida agraria de las poblaciones de Elche y Crevillente; como es el caso de los caballos, yeguas, burros y, sobre todo, mulas, utilizados como animales de tiro. Otros animales cita. 
dos son las cabras, gallinas, pollos, conejos, cerdos..., cuyo claro aprovechamiento sería el de alimento de la población.

No es fácil conocer el lugar ocupado por los animales dentro de la empresa agrícola. Unicamente se podría evaluar su peso dentro del total gracias a las escrituras de inventarios, cartas nupciales y repartos de herencia, pero las menciones en ellas no son demasiado abundantes. No obstante, para hacernos una idea de lo que podría ser su peso específico, que seria importante, podemos citar el caso de Josefa Mellado(25), que entre los bienes que aportó a su matrimonio destacan dos mulas, dos yeguas, dos caballos, un potro, una burra vieja, cuatro cerdos y unas gallinas, cuyo monto asciende a 2.990 reales que, comparado con los 6.272 reales del total de la dote, supone un $47,67 \%$.

2.-Industria: Las actividades industriales o de carácter manual no son referidas con profundidad en las escrituras analizadas. En primer lugar, no existe ningún contrato y la organización interna del mismo no queda reflejada en la documentación notarial. Además está el problema de la no constatación de la profesión de los protagonistas del acto jurídico, y en cuanto a las técnicas industriales utilizadas las noticias son muy escasas, limitándose a las referencias hechas sobre algunas herramientas o instrumentos.

Las actividades registradas, fundamentalmente gracias a las obligaciones, cartas de pago y arrendamientos urbanos, son: la construcción, la fabricación de esteras y la extracción de sosa y sal, en el caso de Crevillente; y las almazaras, molinos y hornos, en Elche. Se sabe también de otras actividades, como las jabonerías y la producción de piedra barrilla, concretamente en Elche, pero aparecen simplemente citadas sin incidir demasiado en sus características(26).

Respecto a la construcción, se trata de obras municipales y sólo en una ocasión se señalan los materiales utilizados: cal de codol, piedra, yeso y hormigón (27); mientras que, como ocurre en las demás actividades, se desconocen el tipo de relaciones laborales y la cualificación profesional de quienes intervienen en los trabajos, sólo podemos establecer la presencia segura de un maestro albañil o alarife y la de un sobrestante(28). Sobre la fabricación de esteras, los protocolos no son más explícitos. Un grupo de unas diez obligaciones y cartas de pago es el que nos habla de la comercialización de las mismas y del junquillo utilizado en su fabricación. Escasas son las revelaciones sobre el carácter de la producción y las técnicas de trabajo(29). En cuanto a la extracción de sosa, la única información concreta es la vinculación de Crevillente con Elche(30), como centro de transformación, y con las Pías Fundaciones, como lugar de extracción(31). 
En el caso ilicitano tenemos los arrendamientos de algunas almazaras, hornos y molinos harineros. La duración de estos arriendos suele ser generalmente, al igual que los rústicos, de unos seis años, salvo para los hornos, cuya duración es de uno o dos años. Igualmente, el monto de la renta es anual y los plazos son semejantes a los arriendos rústicos, a excepción de molinos y hornos, indicándose entonces la renta diaria y los pagos por semanas o meses vencidos(32). Hay que señalar que el arrendamiento de un mismo horno o molino después de pasado un tiempo, permite conocer la evolución seguida por el precio de dicho arriendo. Asi, tenemos que don Ramón Miralles de Miralles cobraba en $1792^{(33)}$ por el arrendamiento de su molino la cantidad de 20 reales diarios a satisfacer por meses vencidos, y en 1799 (34) al mismo arrendatario y con las mismas condiciones le sigue cobrando dichos 20 reales diarios. En cambio, don Benito de Santacilia, que daba en arriendo su horno por el precio de 6 libras y 10 sueldos mensuales en 1792(35), cobrará por el mismo, pero en 1800(36), 8 libras, 6 sueldos y 8 dineros al mes; igualmente, doña Hipólita Tárrega cobrará 5 reales y 12 dineros diarios por su horno arrendado en $1797^{(37)}$, aumentando para 1798(38) en 6 reales diarios.

Ya hemos dicho que son escasas las noticias sobre las técnicas industriales, limitándose a detallar algunos instrumentos. En este sentido, el arrendamiento de un molino harinero(39) describe los siguientes enseres:

«... 6 dedos y tres tercios de la muela corredera a 40 rls.; 8 dedos la Solera, 320 rls.; dos sercoles de hierro en dichas piedras, 130 rls.; cinco sercoles de la otra rueda y alzador, 44 rls.; cuatro picos y dos martillos, 38 ris.; una suela y escoplo, 5 rls.; paleta, barra y un perpal, 52 rls.; el bronce de uso, 30 rls.; la rueda de madera, 160 rls.; banco y alzador, 40 rls.; una botana, 6 rls.; la tolva, 16 rls.; dos barras de volver la muela, 6 rls.; y cuatro congrells, 10 ris..."

En cuanto a las condiciones de estos arrendamientos, podemos señalar como más particulares las que hacen referencia sobre todo a molinos y almazaras. En el caso de los molinos $(40)$ se especifica que siempre que el propietario tiene molienda franca, los gastos del molino serán a expensas del arrendatario, el gasto de levantar las «saetias" será a medias, etc.; y en las almazaras(41), los arrendatarios han de pagar el importe de las piezas que rompan, pero si éste sobrepasa los 10 reales será de cargo del dueño.

Los propietarios de estos medios de producción suelen pertenecer a la pequeña nobleza - don Ramón Miralles, don Benito de Santacilia y miembros de la familia Perpiñán- y a las clases medias urbanas.

3.-Comercio: De las escrituras básicas para su estudio destacamos las cartas de pagos y las obligaciones. Estas nos demuestran que las actividades mercantiles de las villas de Crevillente y Elche habian alcanzado, 
sobre todo en esta última, cierto grado de diversificación. En este sentido, nos encontramos con numerosas escrituras donde la cantidad satisfecha o a satisfacer procede del valor de la venta de animales relacionados con la vida agraria de la comarca, como es el caso de los caballos, asnos y, sobre todo, cabras (42) y mulas (43). En segundo lugar, tenemos la compraventa de productos agrícolas: aceite(44), trigo, cebada, higos, algarrobas, etc. $Y$, por último, aparecen diversos productos de importación que revelan el grado de desarrollo comercial alcanzado, sobre todo por Elche, y cómo el nivel de vida de la clase media va aumentando. Así, por ejemplo, se traía de fuera el cacao de Caracas (45), la canela y pimienta fina de Holanda(46), lino de Francia(47), azafrán, azúcar, cardenillo o verdete(48), hierro, tabaco, ropa, arroz...

La documentación presenta una serie de dificultades que impide conocer con certeza a los comerciantes de Crevillente. En cambio, respecto a Eiche cabe destacar, entre los comerciantes más citados, en primer lugar, a don Antonio de La Murere, capitán retirado agregado a la plaza de la ciudad de Alicante y vecino de Elche, cuya actividad comercial es elevada y se concentra en determinados momentos: en agosto de 1787, con la venta de mulas y cebada; en enero de 1791, la de hierro, y en los meses de febrero y marzo del mismo año, la de cacao y hierro; probablemente estuviese en relación con alguna compañia que trajera estos productos, de ahi la concentración de sus ventas en determinadas fechas. Otros comerciantes serian: Pedro Yrles de Boix, por sus ventas de mulas; Josef Galvis de Mas, por sus ventas de todo género de ropas, y Leonardo Lopes de Paya, por sus ventas de ropa y productos como azafrán, pimienta, lino, canela y azúcar.

Las escrituras de $\mathrm{J}$. Ardid evidencian el mantenimiento de relaciones comerciales entre Crevillente y Alicante, ésta última como centro de abastecimiento de una serie de productos. No obstante, la información con la que contamos es muy fraccionaria, no mostrando la continuidad de esta vinculación. Igualmente, los protocolos de P. Pasqual señalan las relaciones comerciales establecidas entre la villa de Elche y los núcleos urbanos próximos, como el caso de Crevillente, Santa Pola y Alicante(49).

Otro aspecto es el de la organización comercial, es decir, la existencia de compañias cuya actividad se desarrolla en la villa. Pero este punto tropieza con la limitación informativa de los protocolos, con lo que habria que recurrir a otro tipo de fuentes. Sólo en una ocasión, en una escritura de poder(50), tenemos noticia sobre la liquidación de una compañia. En ella se hace referencia a diversos productos - barrilla dulce y salada, sosa, salicor, mesela y demás herbales, también cominos y otros géneros y especias - que fueron vendidos por la compañia a diferentes comerciantes de la ciudad de Alicante y a otras personas, y también las compras efectuadas por dicha compañia. 
Debemos terminar diciendo que el estudio de las cartas de pago y de las obligaciones permite realizar análisis sobre la coyuntura, la situación económica general y el nivel del endeudamiento popular, ya que nos dan a conocer los nombres tanto de acreedores como de deudores, su nivel social y la cuantía de las deudas contraidas (51).

\section{Sociedad}

Este segundo gran bloque lo podemos desarrollar en dos ejes principales:

1.-El régimen matrimonial y la institución familiar: Para su estudio son necesarias las cartas nupciales o recibos de dote y los testimonios. En las zonas donde estas escrituras sean abundantes y estén minuciosamente detalladas, como en el caso francés, se puede observar como bien dice A. Eiras Roel (52):

"... la procedencia social y las posibilidades económicas de las parejas de contrayentes, la correspondencia entre niveles económicos y relaciones sociales, la distribución de los grupos socioprofesionales y la repartición de las fortunas, la naturaleza y origen -y a veces el empleo- de los capitales, la movilidad social y geográfica de los esposos, la psicología social en aspectos como la importancia de la mujer según épocas y niveles de posición social, las proporciones de la endogamia sociológica en una sociedad tradicional o de las relaciones matrimoniales entre grupos sociales próximos..., la evolución en el tiempo del régimen legal del matrimonio, régimen dotal, separación de bienes, comunidad universal u otros...".

De ahí que aplicaciones prácticas de los contratos matrimoniales para el estudio de las sociedades sean abundantes entre los historiadores franceses, debido a su elevado número y detalle, y en relación con el sistema del régimen matrimonial francés que hace necesarias estas escrituras para garantizar la separación de bienes y su disponibilidad por cada uno de los contrayentes. En cambio, este tipo de escritura no es tan abundante en los fondos notariales españoles debido a que aqui nos encontramos ante un régimen matrimonial caracterizado por un régimen dotal con la separación de bienes propios de cada uno de los cónyuges.

Nuestra documentación sólo nos proporciona el nombre, apellidos, estado civil y vecindad de los conyuges y de sus padres; pero no se alude a la profesión -dato fundamental-, ni al lugar de nacimiento. De ahí que interesantes aspectos dentro de este campo no sea posible desarrollarlos por la escasez de noticias ofrecidas; de todos módos, señalaremos algunas conclusiones. 
En las fórmulas legales de las cartas nupciales no se hace ninguna mención directa a la celebración de la ceremonia, incluso en ocasiones la escritura se realiza después de algunos años de matrimonio. En las protocolizadas por J. Ardid es difícil concretar si el matrimonio responde a un convenio previo entre las dos familias, puesto que no sabemos si las expresiones que se utilizan - "... ambas partes combinieron que..."- responden a un hecho real o son simple terminología notarial. Unicamente se tienen algunos indicios de la existencia de una formalización previa; éste es el caso de una renuncia de palabra de casamiento(53). Por otra parte, en las escrituras de P. Pasqual, la carta nupcial se presenta a manera de contrato entre las familias de los contrayentes:

“... parecieron de una parte Xavier Canals y Rosalea Macia, consortes, y de otra Pedro Pasqual y Gertrudis Martinez, tambien consortes, todos vecinos de esta villa... y dijeron: que por tanto tienen tratado y contratado que Josef Pasqual y Martinez y Maria Antonia Canals y Macia casen con la bendición...."(54).

Confirmado esto, tenemos una revalidación de la palabra de casamiento(55), en la que la otorgante se separa de la acción de demanda que tenía interpuesta contra el otorgante, con la plena confianza de que cumplirá con lo prometido y revalidado.

En lo referente al régimen de bienes del matrimonio, ya hemos dicho que se caracteriza por la separación de bienes. Así, en las cartas nupciales, y también en los testamentos y repartos de herencia, se observa una escrupulosidad en la diferenciación de los bienes de cada cónyuge.

Muy poco es lo que se puede decir sobre la procedencia de los cónyuges. Prácticamente, la totalidad de los matrimonios se conciertan entre vecinos, bien de la villa de Elche o de Crevillente. Son muy escasos los enlaces con forasteros $(56)$.

Teniendo en cuenta que no se precisa más que aisladamente la profesión de los cónyuges y de los padres, es imposible un profundo análisis de las alianzas matrimoniales o de las distintas jerarquías sociales en función de las cantidades expresadas en las cartas nupciales. Aunque observando la relación de tierras de cultivo, de aperos y de animales, podemos afirmar que la mayoria de estas escrituras pertenecia al estrato social de los campesinos, tanto pequeños como medianos cultivadores.

A falta de estos datos, nos limitaremos a dar una visión de conjunto de las cuantías de dotes y capitales. De entrada, debemos hacer constar la diferencia numérica existente entre las cartas nupciales y recibos de dote escriturados por P. Pasqual -29 en total - y los de J. Ardid -128-. En éste último, el $50 \%$ de los capitales se mantiene por debajo de los 1.000 reales, y de ese porcentaje, el $57 \%$ no supera los 500 rls.; en el lado opuesto, sólo el $15 \%$ sobrepasa los 2.500 rls., y el 35 restante se encuentra entre los 
1.000 y 2.500 rls. Por su parte, el $77 \%$ de las dotes no supera los 1.000 rls., $y$ solo el $7 \%$ sobrepasa los 2.000 rls. Comparando dotes y capital, ambos se mantienen a su mismo nivel en las cartas que no superan los 1.000 ris. de capital; en cambio, en donde se superan los $1.000 \mathrm{rls}$. de capital, la dote queda, salvo excepciones, por debajo de esa cifra. Por ejemplo, entre los 1.000 y $2.000 \mathrm{rls}$., las dotes se situan en torno a los $752 \mathrm{rls}$. de media, pero con grandes oscilaciones, que van de dotes de 240 a $2.000 \mathrm{r} / \mathrm{s}$.

En cuanto a las protocolizadas por $P$. Pasqual se puede concluir, de modo ilustrativo, que once dotes presentan cifras inferiores a $1.000 \mathrm{rls}$., nueve tienen totales de 1.000 a 2.000 rls., y seis están encuadradas entre los 2.000 y $3.000 \mathrm{rls}$. De los ocho capitales catalogados, cinco presentan cantidades menores de $1.000 \mathrm{rls}$. y uno sobrepasa escasamente esta cantidad. En cualquier caso, las escrituras que expresan un nivel economico más elevado son minoria. Asi nos encontramos con sólo dos dotes que llegan a superar con poco los 6.000 Is. (57) y dos capitales, uno que llega a los $4.000 \mathrm{rls}$. y otro que sobrepasa los $7.500 \mathrm{rls}$. (58).

Referente a las cantidades en que se realiza la alianza matrimonial, siempre aparece aquella en la que el varón se obliga a tener los bienes de la dote y restituirlos a su esposa en caso de que el matrimonio fuera disuelto por cualquier causa, y además no obliga a su mujer en sus deudas pero si la admite en sus ganancias.

Temas como la exogamia, la endogamia y los matrimonios consanguineos no pueden ser constatados por la insuficiencia de datos y solo seria posible mediante la reconstrucción de familias en base a documentación parroquial. Si podemos afirmar que la pequeña nobleza ilicitana practicaba la endogamia, procurando casar a sus hijos con los de otras familias nobles. Esto queda reflejado en cuatro licencias de matrimonio concedidas por nobles: don Ramón Miralles y dona Antonia Miralles a su hijo don Francisco de Paula Perpiñan (59); don Pascual Ortis Martinez de Vera y Almodovar a su hijo don Rafael Ortis de Almodovar (60); doña Francisca Antonia Vaillo de Llanos, viuda, a su hijo don Francisco Xavier Sarabia(61), y don Antonio de La Murere a su sobrino don Manuel Lamaur(62). En ellas se observan las uniones nobiliarias con lo que ello significa de unión de apellidos y titulos.

Estos mismos documentos son los que nos informan sobre la familia. A partir de los testamentos es posible conocer el número de hijos del matrimonio, los habidos en anteriores nupcias, los fallecidos e, incluso, el nacimiento de algún hijo póstumo(63).

La composición de la familia es muy variable, oscilando entre las parejas que declaran no tener sucesion y los once hijos de Gaspar Martines 
en sus tres nupcias(64) o los tréce de Jayme Campello en sus dos matrimo. nios (65). Entre estas cifras extremas, son los cinco, cuatro y seis hijos, por este orden, las que más se repiten en los testamentos crevillentinos; en los ilicitanos predominarán las de uno, seis, cinco y dos. En algún caso excepcional, la pareja sin hijos acepta a algún menor de edad, al que crian como hijo (66). Incluso hemos recogido una escritura de prohijacion que formaliza esta adopción (67).

Al margen de los conyuges y sus hijos es dificil concretar la convivencia de otros miembros familiares o no en la misma casa. Asi, se citan nietos, sobrinos y también criados ${ }^{(68)}$ de los testantes, a quienes se les concede algún legado, aunque de este dato no se puede deducir si vivian junto con la pareja; más bien se trata de una muestra de afecto.

Otras veces se pone de relieve una estrecha dependencia entre algu. no de los componentes del clan familiar, aunque no se aclara si todos habitan juntos ${ }^{(69)}$. Solo aisladamente, en situaciones concretas, se puede observar con claridad la permanencia en un mismo hogar de algún pariente proximo(70). Ciertas cartas nupciales sí nos confirman la residencia de los padres con algunos de sus hijos. De modo que los padres conceden una casa a sus hijos, imponiendo como condicion su convivencia con la nueva pareja(71). A veces este mismo hecho se manifiesta sin mediar ninguna condición, con la concesión de los padres de parte de la casa en que viven - suponemos que los recién casados residirán con ellos, puesto que casi nunca se habla de la existencia de otra vivienda propia.

En suma, si bien a partir de estos datos es dificil conocer con certeza la estructura de la familia, como algunos autores han podido hacer basán. dose en las cartas nupciales(72), si podemos llegar a alguna conclusión ayudándonos de los testamentos, aun a riesgo de que sea muy eventual. El reparto que el padre hace de sus tierras entre los hijos nos lleva a pensar que la familia amplia, polinuclear, tiene poca razón de ser, puesto que cada hijo del matrimonio conserva su propiedad independientemente de los demás. Como mucho, es la madre, y a veces el padre, quien vive junto con alguno de sus hijos casados, compensándole con la donación de una propiedad, generalmente la propia vivienda. En cualquier caso, existe la organización patriarcal de la familia, reconocida como principio del derecho común, que hace del hombre el cabeza de familia, reconociéndose su jefatura. Igualmente el papel de la mujer es considerado inferior $y$, a veces, suplementario, al del hombre; además, habria que tener en cuenta su condición social y civil(73).

2.-Estratificación social: De entrada, debemos señalar que nos vamos a encontrar con el problema de la no constatación, en la mayoría de las escrituras, de la profesión de los protagonistas del acto jurídico. De ahí que resulte dificil analizar los distintos grupos sociales, las relaciones entre ellos y el peso específico de cada grupo en el conjunto de la comunidad. 
En primer lugar, tenemos las referencias a la nobleza. En la documentación de J. Ardid, estas referencias son escasas, por no decir nulas. El estrato social más alto que aparece en las escrituras crevillentinas corresponde a los administradores señoriales: el Administrador de Rentas (74), el Juez de Residencia (75) y el Administrador de la villa(76). En este nivel uno de los personajes más destacados es el doctor Phelipe Sempere, abogado de los Reales Concejos y Gobernador de Crevillente(77), que en octubre de 1740 tomaba posesión del señorio de Elche en representación de don Manuel Ponce de León, Duque de Arcos.

Los protocolos de P. Pasqual ofrecen mucha más información sobre el estamento nobiliario de la villa de Elche. Se nos habla de los diversos cargos municipales y de quiénes los detentan: don Pasqual Ortis Martines de Vera y Almodovar, como Síndico Personero del Público(78); don Josef Manuel Sanches del Aguila, Alcalde Mayor(79); don Felix Despla y don Manuel Urtasun, alcaldes primeros ordinarios(80); don Gaspar Melendes y Miralles, alcalde segundo(81); y otros como regidor tercero, segundo diputado de abastos, etc. De diferentes títulos nobiliarios: don Francisco Soler de Cornellá, señor Caballero Maestrante de la Real Maestranza de la ciudad de Valencia(82); don Benito Ruiz, Caballero Maestrante de la Real de Ronda(83); don Joaquín Perpiñán y Pasqual (84) y su hijo don Joaquín Perpiñán y Tarrega ${ }^{(85)}$, Caballeros del Hábito de Nuestra Señora de Montesa y San Jorge de Aljama, entre otros.

A finales del siglo XVIII la pequeña nobleza supo promocionarse militarmente $y$ adquirir, en consecuencia, un considerable poder: las milicias urbanas, los voluntarios honrados, las milicias provinciales... Pocos eran los nobles ilicitanos que no gozaban, como uno más de sus privilegios, del título de comandante, capitán u otro semejante, de milicias, con su "fuero militar» incluido. En 1794, la milicia urbana cambia en Elche su nombre por el de "compañía de voluntarios honrados", más en consonancia con el carácter paramilitar que la guerra contra Francia impone(86). Podemos destacar los siguientes: don Pedro Miralles de Ymperial, capitán de una compañía de voluntarios honrados (87); don Francisco de Paula Juan, capitán(88); don Manuel Urtasun, teniente(89); don Joaquín Perpiñán y Tarrega, sargento mayor y comandante interino del batallón de voluntarios honrados (90); etc. También se detallan otros cargos militares: don Máximo Miralles de Ymperial, teniente del regimiento de las cuatro Ordenes Militares (91); don Antonio de La Murere, capitán retirado(92); don Gerónimo Martin Cortes, teniente coronel retirado y gobernador del castillo de Santa Pola(93); don Josef Zaravia Vaillo de Llanos, teniente coronel retirado(94); don Francisco Xavier Sarabia, alférez de navío(95), entre los más citados. 
En cuanto al clero, aparece representado, en Crevillente, por los sacerdotes de la iglesia parroquial de la villa. Los únicos cargos destacados que se citan son: el doctor don Ambrozio Guillem, presbitero rector (96), y el doctor Joseph Mas, presbitero calificador del Santo Oficio(97). En los protocolos de P. Pasqual son muy escasas las noticias sobre el clero; tan sólo tenemos unas pocas escrituras realizadas por nuestro escribano en la Sala de la encomienda del convento de la Real y Militar Orden de Nuestra Señora de la Merced, algunas referencias a presbiteros, a la entrada de nuevos religiosos ${ }^{(98)}$ y a algunos familiares del Santo Oficio.

También están representados los miembros de profesiones liberales. Esencialmente encontramos en este grupo a los abogados de los Reales Concejos, a los escribanos y a los amanuenses, que trabajan en cada villa. Intervienen con mucha frecuencia en operaciones comerciales, como arriendos, ventas y préstamos, y también en las escrituras de poderes. En Crevillente destacamos al doctor don Josef Prefacio; y en Elche a don Gaspar Esclapez que actúa muchas veces de apoderado, sobre todo de don Pedro Leguey. Al margen de ellos, sólo de forma excepcional se citan otras profesiones: médicos(99), cirujanos(100), maestros de escuela(101), estanquero de tabacos y administrador de la real renta de Tabacos(102), subarrendatario del real derecho de nieve e interventor de la renta de naipes (103), fiel de terrena(104)...

Aparte de estos ejemplos, la mayor parte de los que acuden al escribano pertenecen a clases medias y bajas, siendo éstos comerciantes, artesanos y campesinos. El problema reside en que en las escrituras no se indican estos términos, salvo excepciones.

Como anteriormente hemos hablado ya de los comerciantes, pasemos ahora a citar algunos ejemplos dentro del grupo de los artesanos. Sobre Crevillente tenemos muy pocos casos: un maestro alarife(105), un herrero que se constituye como capital las herramientas y lo que le costó el examen del oficio(106) y un carpintero que igualmente se adjudica sus útiles de trabajo(107). Es en los inventarios de P. Pasqual donde se encuentran referencias a estas actividades, ya que en ellos se nombra una serie de peritos para valorar los distintos bienes. Asi tenemos a maestros agrimensores y expertos labradores(108), maestro albañil, carpintero y herrero(109), costurera (110)... No hay que olvidar los que trabajan en los molinos, almazaras, hornos, etc., actividades de las que ya hemos hablado en otro apartado.

Pero la mayor parte de los que acuden a ambos notarios son pequeños y medianos campesinos, tanto cultivadores como propietarios. Aunque no se especifique esta actividad, basta observar en las escrituras las numerosísimas referencias a todo lo concerniente a la agricultura, tanto tierras como productos y bienes, y es que nos encontramos con núcleos urbanos que desarrollan una vida rural. 
Por último, cabe indicar que las escrituras nos pueden detallar sucesos o conflictos sociales acaecidos en la zona. Este es el caso del poder que don Pasqual Ortis Martinez de Vera y Almódovar, Síndico Personero del Público de Elche, otorga a don Miguel de Monasterio, don Antonio de Pargas y don Ignacio Machuna para que se presenten en el Real y Supremo Consejo de Castilla y se opongan a la Insaculación practicada para los gobernantes de la villa de Elche en el mes de febrero de 1788 y para que soliciten el nombramiento de nuevos oficiales de Justicia y Gobierno(111).

Más relevantes aún son las tres protestas(112), recogidas por el escribano J. Ardid, en las que se refleja con claridad la extensión que tuvo por la geografia española, en este caso en Crevillente, el motín de Esquilache que estalló el 23 de marzo de 1766 -Domingo de Ramos - en Madrid. En ellas se observa, después de los disturbios, el restablecimiento de la calma a finales del mes de abril. Los declarantes, entre ellos el mismo escribano, se manifiestan en favor del Rey y del Duque de Arcos frente a los amotinados.

\section{Cultura}

En primer lugar, trataremos la cultura material. La determinación de la naturaleza de las fortunas es un factor tan importante o más que la definición de los montantes cuando se pretende realizar una clasificación de carácter social(113). Y aún más se ha de tener en cuenta, como sugiere $R$. Mousnier(114), el conocimiento de los modos y estilo de vida de los distintos estratos de la población. Estos aspectos encuentran en las cartas nupciales e inventarios su principal fuente de estudio. Estas escrituras, al relacionar los bienes muebles e inmuebles de los protagonistas del acto notarial y señalar, al mismo tiempo, el valor de cada uno de ellos, se convierten en una fuente indispensable para el estudio de las condiciones y formas de vida de la población, ilustrándonos sobre cuáles son sus ropas, sus muebles, sus ajuares domésticos, sus útiles de trabajo, sus joyas, sus propiedades rústicas y urbanas..., permitiéndonos establecer diferencias de fortuna y de confort de los diversos grupos sociales. Pero estas diversas posibilidades se ven limitadas, en cierta manera, por su escaso número. De todas formas, su información no es nada desdeñable, puediendo llegar a conocer más de cerca cómo se desenvolvía la vida cotidiana y en qué condiciones, así como la cultura material de la época, siendo en los casos de Elche y Crevillente la propia de un núcleo urbano que desarrolla una vida rural tradicional.

Otro dato muy significativo, ya sobre el nivel cultural de una época, es la presencia o ausencia de libros y bibliotecas. En nuestro caso particular, no ha sido repertoriada ninguna biblioteca ni la posesión de libros por particulares. Sin embargo, hay autores que han realizado numerosos trabajos 
sobre esta cuestión gracias a que contaban con una descripción minuciosa de los libros poseídos(115).

Otro punto es el nivel de alfabetización de la población, analizado por algunos autores (116) a través de las firmas y noticias aparecidas en las escrituras.

En este campo cultural hemos dejado para el final el importantísimo tema de la realización de una historia de las mentalidades, fundamentalmente, en su aspecto religioso. La principal, y se puede decir también exclusiva, fuente notarial serían los testamentos. De entrada, se describiría el ritual fúnebre con todas sus prácticas, como la invocación, el lugar del entierro, el hábito elegido, las mandas de misa y de limosna. Con ello, se ahondaría en las creencias religiosas, siguiendo el proceso secularizador o "descristianizador" y conociendo una serie de valores espirituales sostenidos por la gente de la época.

Un primer punto a tratar es el de la condición física del testante. Tenemos así que un $70 \%$ de los testantes señalan su condición de enfermos, lo cual puede llevar a la conclusión de que una cierta mayoría de la gente esperaba a dejar constancia de su última voluntad en las postrimerías de su vida. En cuanto a la representatividad por sexo se ha obtenido un perfil casi equilibrado entre testantes maculinos y femeninos.

La invocación presenta como rasgo importante su uniformidad, la ausencia de variación formal, lo cual permite afirmar que su contenido responde a una fórmula notarial estereotipada que cada escribano aplica a sus actas. Pero, de todos modos, estas fórmulas introductorias mantienen una relación con la vida y el pensamiento de la sociedad. En todas las ocasiones se menciona expresamente la creencia en el misterio de la Trinidad y se ruega la intercesión de la Virgen María ante Cristo, para obtener de El el perdón de los pecados y el eterno descanso para el alma. De todos los testamentos escriturados por ambos escribanos, sólo en una ocasión hay un ruego a un santo, concretamente, San Juan Bautista(117).

Por otro lado, la casi totalidad de los testantes, tanto hombres como mujeres, piden que su cuerpo sea revestido con el hábito de San Francisco, cuya intercesión se considera una garantía de salvación. Ahora bien, en los protocolos de J. Ardid se han contabilizado unos cuatro testantes y en los de P. Pasqual unos siete que eligen otro hábito(118). En el primer escribano se expresa de forma aislada el interés por el enterramiento con ataúd, coincidiendo siempre con las escrituras en las que el ritual funerario es más importante, lo que hace pensar que esta práctica no era norma general. En cambio, en P. Pasqual se repite con frecuencia la petición de ser enterrado con ataúd, incluso a veces se especifica que esté forrado de bayeta negra(119). 
La sepultura se fija siempre en la iglesia parroquial de donde el testante es feligrés: parroquia de Crevillente y las de Santa Maria, El Salvador y San Juan de Elche; también en algunas capillas de conventos. Es de senalar que todavia no habian surgido los cementerios extramuros. Respecto a Elche, pasada la epidemia del año 1811 se pensó en construir un cementerio extramuros, según lo mandado en las circulares de 1804 y la orden superior que en cabildo celebrado el 17 de noviembre de 1809 se cumplimento, en que se ordenaba por la Suprema Junta Central la construcción de cementerios rurales siendo terminado en $1812^{(120)}$. Otro aspecto es que algunos nobles y burgueses son propietarios de sepulturas dentro de dichas iglesias o en conventos(121).

En lo que concierne al ritual del entierro, la simplicidad es la nota dominante; generalmente se desea acompañamiento ordinario con asistencia de la cruz de la parroquia. Son escasas las peticiones de un ceremonial más complicado, deseando en esos casos ser acompañados por los eclesiásticos residentes, porcionistas o asistentes de la iglesia parroquial, o piden la presencia de representantes de distintas corporaciones o conventos. De este modo, Ignacio Galvañ señala que le han de acompañar, además de los residentes, la comunidad de San Francisco, la de San José de Elche y la de los carmelitas calzados de Cox(122). Por su parte, don Josef Mendiola pide que su féretro sea conducido por seis religiosos del convento de San José y que la procesión de su entierro sea pobre, reduciéndose los residentes de tanda, concurriendo las tres cruces de las parroquias de Elche y con acompañamiento de doce pobres con antorchas (123).

Después de las disposiciones sobre el cuerpo se desarrollan las que atañen al alma, en concreto misas y limosnas. Una vía para el estudio del proceso secularizador o "descristianizador" de la sociedad es ir analizando la evolución que sigue el número y clase de misas y las limosnas que se dan, a través del vaciado de todos los testamentos del siglo XVIII y mediados del XIX. Con nuestra reducida y parcial muestra resulta difícil poder extraer alguna conclusión clara respecto a la evolución de la mentalidad religiosa a través de las misas. Podemos señalar que en los testantes crevillentinos predominan las mandas de menos de 50 misas, y en los ilicitanos un $72 \%$ realiza mandas hasta un número de 100 misas; existen también algunos casos en donde las misas son bastantes numerosas (124). La limosna habitual por cada misa era de cuatro reales o una peseta, siendo raros otros pagos, y en algunas ocasiones, como ocurre con casi todos los testamentos de J. Ardid, no se especifica, lo cual parece indicar que tenian ya un precio estipulado. 
Todas estas misas eran repartidas según la devoción del testante, mostrando especial interés en que se celebren parte de ellas en la parroquia donde cada cual desea ser enterrado y las restantes entre las otras parroquias, conventos y capillas, destacando en este sentido la de San Juan de Letrán del convento de Nuestra Señora de la Merced de Elche, que se consideraria como una garantia de salvación. También se reparten en centros de culto pertenecientes a otras localidades, como Almoradi, Monforte... No hay que olvidar el gran número de misas que los testantes dejan a elección de sus albaceas.

Igualmente interesa tener en cuenta el número de misas perpetuas y de cuerpo presente, aspecto que presenta gran diferencia entre los protocolos de J. Ardid y de P. Pasqual. En el primero, sólo tres veces se establece el total de misas de cuerpo presente que se han de celebrar; por su parte, el porcentaje de misas perpetuas no es muy superior, puesto que únicamente aparecen en cuatro ocasiones, junto con otros dos testamentos en los que se instaura una dobla anual(125), asignando siempre los pagos anuales por las mismas. De las cuatro misas perpetuas, una, a celebrar el día de la Purisima, se acompaña de un rosario(126), y otra se presenta como una misa de aurora a celebrar el día 1 de enero de cada año(127). Por el contrario, los testantes que acuden a $P$. Pasqual piden siempre la celebración de una misa de cuerpo presente, pero no hemos encontrado ninguna manda de misa perpetua, tan sólo la petición de un diario de misas rezadas hasta donde llegue el dinero donado(128). Como se puede observar, conforme transcurre el siglo XVIII se van abandonando las misas perpetuas, siendo sustituidas por las misas sueltas.

Otro punto interesante es el conocer el concepto de la caridad de la época. En J. Ardid, las limosnas ofrecen siempre un punto común: todas son destinadas a la Casa Santa de Jerusalén, y excepcionalmente se realizan donaciones al Santo Hospital y Redención de Cautivos. En todos los testamentos de P. Pasqual se repiten las limosnas, con diferente valor, a la Casa Santa de Jerusalén, Pobres Cautivos Cristianos y Santo Hospital de la villa de Elche. Esta repetición de las mandas caritativas nos hace pensar que en este sentido estaban estrechamente unidos el espiritu religioso y la apariencia social, y conectado todo con el formulismo notarial. Al margen de estas donaciones generales, otras limosnas se concedian al sacerdoteque asiste al testante antes de morir, algún lugar o imagen de especial devocion(129), alguna hermandad(130), a pobres(131)...

Una vez que se han resuelto los problemas espirituales, se lleva a cabo la parte referida a los bienes y problemas terrenales. Este aspecto, con la secularización de la sociedad, irá creciendo en cuanto a espacio y precision, relegando lo religioso y espiritual del testamento a un claro segundo término y convirtiéndose al final en un acta juridica de carácter estrictamente civil. El estudio de esta evolución necesitaria, como ya hemos 
dicho, el vaciado de los testamentos, sobre todo del siglo XVIII y mediados del siglo XIX.

En esta linea existen importantes trabajos franceses, como los de Ph. Ariès(132), Ph. Goujard(133), F. Lebrun(134), P. Chaunu(135), pero sobre todo destacan los estudios realizados por $M$. Vovelle(136). Este distingue las siguientes etapas: 1680-1710, momento de mayor esplendor de las pompas barrocas; 1710-1740, fase ambigua en la que la media de misas baja sensiblemente; 1740-1760, el declive es continuo, observándose en las misas de fundacion; y 1760-1790, con un testamento casi totalmente laicizado en sus formulas. Vovelle concluye diciendo que podría afirmarse que datar el retroceso de demandas de misas, de cambio de sepultura, de cambio de formulario testamental, no es datar el retroceso de la piedad. De ahi que clarifique que la piedad colectiva cambia de hábitos pero no de contenido. Es decir, ninguna de estas mutaciones da una seguridad suficiente de descristianización en el Siglo de las Luces, pero parece converger en la cronologia de su evolución. El caso es que se produce un cambio más amplio y la descristianización no es más que uno de sus aspectos: para los provenzales del siglo XVIII la imagen de la muerte ha cambiado.

Destaquemos, por último, el trabajo del español Baudilio Barreiro Mallon (137) que llega a resultados diferentes a los obtenidos por los autores franceses, como F. Lebrun y $\mathrm{M}$. Vovelle, los cuales indican para algunas zonas francesas un cambio en el sentido laicizante a partir de 1760 . En cambio, B. Barreiro, siguiendo el método de contrastar los datos obtenidos de los Archivos parroquiales que tengan sus libros de defunciones con los procedentes de los testamentos, señala que el pueblo gallego mantiene la misma linea de espiritualidad desde mediados del siglo XVII hasta primeros del XIX; solo observa dos cambios: la desaparición de las ofrendas y el abandono de las misas de fundación, sustituidas por las misas sueltas.

A nuestro juicio, los protocolos que hemos estudiado parecen indicar que la religiosidad vivida en la zona de Elche se asemeja más a la del pueblo gallego que a la señalada por los historiadores franceses. 


\section{NOTAS}

(1) F. FURET: “Histoire quantitative et fait historique». En Annales, 1971, número 1, pp. 63 75. Llamadas por el autor como afuentes no estructuralmente numericas, pero sin embargo seriales y fácilmente cuantificables" (pp. 70-71).

(2) Antonio EIRAS ROEL y colaboradores: La Historia social de Galicia en sus fuentes de protocolo. Secretario de Publicaciones de la Universidad de Santiago de Compostela, 1981. 564 pp.

Ofrece una amplia muestra de las aplicaciones que han tenido los protocolos en los campos de la historia rural, urbana y de las mentalidades.

(3) Pedro F. JOVER IBARRA: Tipologia notarial del Archivo Municipal de Elche en el siglo XVIII: protocolos del escribano don Pedro Pasqual de Ganga (1786-1800). Tesis de Licenciatura. Facultad de Filosofia y Letras, Universidad de Alicante.

(4) Rafael PLA GRAU: Protocolos de Joseph Ardid (1735-1780): una aproximación a la tipologia documental de los protocolos del Archivo Municipal de Elche. Tesis de Licenciatura. Facultad de Filosofia y Letras, Universidad de Alicante.

(5) Por citar un ejemplo reciente, éste ha sido el esquema seguido en la distribución de las comunicaciones en el If Coloquio de Metodologia Histórica Aplicada, con la particularidad de que se dedica un apartado especial a la historia rural. Ver: Resumen de las Comunicaciones del 1/ Coloquio de Metodologia Histórica Aplicada. Celebrado del 27 de septiembre al 1 de octubre de 1982. Facultad de Geografia e Historia. Departamento de Historia Moderna. Universidad de Santiago de Compostela. 106 pp.

(6) Antonio GIL OLCINA: "El regadio en Elche». En Estudios Geográficos, n. 112-113. pp. 527-574. En la pág. 552 senala los datos recogidos por Cavanilles sobre las principales producciones del campo ilicitano.

(7) Guy CABOURDIN: Terre et hommes en Lorraine, 1550-1635. Universidad de Nancy, 1977. Dos volúmenes. En este sentido, el autor elabora un mapa sobre la vid, pp. 655-657.

(8) A. M. E. protocolos de Pedro Pasqual, signatura 1148, protocolo 1386 (1799-X-27).

(9) A. M. E. protocolos de Joseph Ardid, signatura 507, protocolo 258 (1741-XI-27).

(10) A. M. E. ps. de P. Pasqual, sig. 591, p. 1269 (1798-III-14).

(11) A. M. E. ps. de J. Ardid, sig. 507, p. 135 (1739-1-11).

(12) A. M. E. ps. de J. Ardid, sig. 509, p. 535 (1754-VIII-29).

(13) Doña Ursula, viuda de don Antonio Tárrega, doña Hipólita, doña Francisca, doña Teresa, doña Mariana, doña Josefa y don Joaquin Perpiñán y Tárrega, caballero del Hábito de Montesa, Sargento Mayor y Comandante Interino del Batallón de Voluntarios Honrados de Estado del Partido de Xixona.

(14) Apoderado de don Pedro Leguey, el cual es administrador de la Excma. Sra. Condesa Duquesa de Benavent y Arcos.

(15) Administrador de don Josef y doña Josefa Perpiñán y Miralles.

(16) Apoderado de don Benito Ruiz de Musoles.

(17) Apoderado de la llustre Sra. doña Mariana Vaillo de Llanos, viuda del llustre Sr. don Antonio Canisia y Pasqual, Marquesa viuda del Bosque.

(18) A. M. E. ps. de P. Pasqual, sig. 1148, p. 1389 (1799-X-29).

(19) A. M. E. ps. de P. Pasqual, sig. 589, p. 676 (1793-VIII-19).

(20) A. M. E. ps. de J. Ardid, sig. 509, p. 520 (1753-VIII-4).

(21) A. M. E. ps. de J. Ardid, sig. 510, p. 826 (1774-VIII-1). 
(22) Pedro RUIZ TORRES: Señores y propietarios. Cambio social en el sur del Pais Valenciano: 1650-1850. Institució "Alfons el Magnànim». Diputació Provincial de València. València, 1981. $403 \mathrm{pp}$.

(23) Antonio GIL OLCINA: La propiedad señorial en tierras valencianas. Edit. Del Cenia al Segura. Valencia, 1979, pp. 35-37.

(24) J. JACQUART: "Sources notariales et histoire rurale». En el Resumen de las Comunicaciones del I/ C. M. H. A. Santiago de Compostela, 1982. pp. $22-25$ (ver pp. 23-24).

(25) A. M. E. ps. de P. Pasqual, sig. 586, p. 124 (1788-1-23).

(26) A. M. E. ps. de P. Pasqual, sig. 588, p. 485 (1791-IV-6). En esta escritura un campanero se obliga a construir un suelo redondo de cobre para caldera de jabón de loza para la jaboneria de Mariana de Montoro.

(27) A. M. E. ps. de J. Ardid, sig. 508, p. 351 (1746-1-30).

(28) A. M. E. ps. de J. Ardid, sig. 508, p. 351 (1746-I-30).

(29) A. M. E. ps. de J. Ardid, sig. 507, p. 163 (1739-VIII-2). Se trata de una carta nupcial en la que señala, entre otros bienes, una rueda con sus útiles e hilo para esteras.

(30) A. M. E. ps. de J. Ardid, sig. 507, p. 121 (1738-VII-17).

(31) A. M. E. ps. de J. Ardid, sig. 508, p. 292 (1743-II-8).

(32) A. M. E. ps. de P. Pasqual, sig. 591, p. 1231 (1797-XII-28). Especifica una renta diaria y un pago semanal cada sábado.

(33) A. M. E. ps. de P. Pasqual, sig. 588, p. 582 (1792-VIII-20).

(34) A. M. E. ps. de P. Pasqual, sig. 1148, p. 1343 (1799-VIII-3).

(35) A. M. E. ps. de P. Pasqual, sig. 588, p. 616 (1792-XII-9).

(36) A. M. E. ps. de P. Pasqual, sig. 1148, p. 1496 (1800-XII-8).

(37) A. M. E. ps. de P. Pasqual, sig. 590, p. 1165 (1796-XII-28).

(38) A. M. E. ps. de P. Pasqual, sig. 591, p. 1231 (1797-XI1-28).

(39) A. M. E. ps. de P. Pasqual, sig. 1148, p. 1373 (1799-X-9).

(40) A. M. E. ps. de P. Pasqual, sig. 588, p. 582 (1792-VIII-20).

(41) A. M. E. ps. de P. Pasqual, sig. 590, p. 1161 (1796-XII-6).

(42) A. M. E. ps. de J. Ardid, sig. 507, p. 74 (1737-VII-7).

(43) A. M. E. ps. de P. Pasqual, sig. 1148, p. 1421 (1800-111-31).

(44) A. M. E. ps. de J. Ardid, sig. 507, p. 223 (1741-II-10).

(45) A. M. E. ps. de P. Pasqual, sig. 588, p. 451 (1791-1l-14).

(46) A. M. E. ps. de P. Pasqual, sig. 590, p. 1130 (1796-IX-3).

(47) A. M. E. ps. de P. Pasqual, sig. 590, p. 1130 (1796-IX-3).

(48) A. M. E. ps. de J. Ardid, sig. 508, p. 357 (1746-V-3).

(49) Pedro RUIZ TORRES: Señores..., op. cit. Siguiendo con el tema, podemos señalar de forma ilustrativa lo que el autor dice en la página 261: “... Había un comercio entre Elche y otras comarcas valencianas (arroz), catalanas (textil) y castellanas (granos). Habia un comercio con el extranjero y con América (barrilla, sosa, aceite). Pero también tenia su pequeña importancia el comercio local: los campesinos llevaban sus productos a la villa; atravesaban sus murallas y pagaban los correspondientes impuestos; a cambio conseguian comestibles y alfareria. En el mercado diario intercambiaban mercancias los artesanos locales y los campesinos...".

(50) A. M. E. ps. de P. Pasqual, sig. 588, p. 567 (1792-VI-18). En este poder se refleja la liquidación de la compañía formada por Manuel López de Serrano y Francisco Bru y Martines, vecinos de Elche.

(51) M. LACAVE: “Crédit à la consommation et conjocture économique: L'lsle-en-Venaisin (1460-1560)». En Annales, 1977, número 6, pp. 1128-1153. Con el estudio de las obligaciones sigue el proceso de la coyuntura económica, la circulación monetaria, la evolución de los precios 
de los productos textiles y de los granos, y el peso del crédito en la economia rural de la zona tratada.

(52) Antonio EIRAS ROEL: “Tipologia documental de los protocolos gallegos". En Historia social de Galicia en sus fuentes de protocolos, de A. Eiras Roel y colaboradores. Secretariado de Publicaciones de la Universidad de Santiago de Compostela, 1981, pp.: 21-113. (Ver pp. 30-31).

(53) A. M. E. ps. de J. Ardid, sig. 510, p. 820 (1774-I-7).

(54) A. M. E. ps. de P. Pasqual, sig. 586, p. 66 (1787-VII-25).

(55) A. M. E. ps. de P. Pasqual, sig. 591, p. 1258 (1798-11-18).

(56) A. M. E. ps. de J. Ardid, sig. 508, p. 299 (1742-IV-22).

(57) A. M. E. ps. de P. Pasqual, sig. 590, p. 1141 (1796-X-3).

(58) A. M. E. ps. de P. Pasqual, sig. 586, p. 108 (1787-XII-25).

(59) A. M. E. ps. de P. Pasqual, sig. 587, p. 320 (1790-IV-9).

(60) A. M. E. ps. de P. Pasqual, sig. 588, p. 457 (1791-II-23).

(61) A. M. E. ps. de P. Pasqual, sig. 591, p. 1308 (1798-VI-18).

(62) A. M. E. ps. de P. Pasqual, sig. 1148, p. 1505 (1800-XII-30).

(63) A. M. E. ps. de J. Ardid, sig. 509, p. 534 (1754-VIII-13).

(64) A. M. E. ps. de J. Ardid, sig. 507, p. 115 (1738-VI-15).

(65) A. M. E. ps. de P. Pasqual, sig. 590, p. 915 (1795-V-19).

(66) A. M. E. ps. de J. Ardid, sig. 509, p. 442 (1750-1-25).

(67) A. M. E. ps. de J. Ardid, sig. 507, p. 70 (1737-VI-9).

(68) A. M. E. ps. de P. Pasqual, sig. 589 , p. 640 (1793-II-21).

(69) A. M. E. ps. de J. Ardid, sig. 510, p. 852 (1780-V-29). Manuela e Isabel Rodriguez, viuda y soltera respectivamente, renuncian a sus derechos a una casa en favor de su hermano Ramón, en atención a que reciben de él "alimento diario y otras utilidades".

(70) A. M. E. ps. de J. Ardid, sig. 507, p. 38 (1736-III-5). La testante Maria Macia concede a su hijo Roque Puig una casa con la condición de que su hija Josepha Maria viva en ella hasta que se case.

(71) A. M. E. ps. de J. Ardid, sig. 507, p. 166 (1738-II-18).

(72) A. POITRINEAU: "De la Haute Auvergne au Bas-Canada: contrats de mariage dans une société "ènraciné"». En Les Actes Notariés. Actas del Coloquio de Estrasburgo. Lib. Istra, 1979, pp. 147-162.

H. MICHEL: «Pratique notarial et société: les contrats de mariage à Montpellier à la veille de la Revocation de l'Edit de Nantes". En Les Actes Notariés. Actas del Coloquio de Estrasburgo. Lib. Istra, 1979, pp. 163-169.

Un ejemplo español es el de A. RODRIGUEZ: «Las cartas de dote en Extremaduram. En Resumen de las Comunicaciones del II C. M. H. A. Santiago de Compostela, 1982, pp. 12-13.

(73) P. PONSOT: "Coup d'œil sur une communauté paysanne bressane auX XVII'-XVIII" siècles d'après les archives notariales". En Les Actes Notariés. Actas del Coloquio de Estrasburgo. Lib. Istra. 1979, pp. 141-145. Este autor descubre a partir de los contratos matrimoniales la estructura de la familia y la condición de la mujer, dependiente de los posibles status: joven soltera, mujer casada y viuda.

(74) A. M. E. ps. de J. Ardid, sig. 510, p. 850 (1779-1X-4).

(75) A. M. E. ps. de J. Ardid, sig. 509, p. 687 (1759-X-2).

(76) A. M. E. ps. de J. Ardid, sig. 510, p. 810 (1767-III-19).

(77) A. M. E. ps. de J. Ardid, sig. 507, p. 248 (1741-IX-2).

(78) A. M. E. ps. de P. Pasqual, sig. 586, p. 130 (1788-11-26).

(79) A. M. E. ps. de P. Pasqual, sig. 590, p. 1104 (1796-VII-11).

(80) A. M. E. ps. de P. Pasqual, sig. 589, p. 724 (1793-XI-24). 
A. M. E. ps. de P. Pasqual, sig. 1148, p. 1348 (1799-VIII-13).

(81) A. M. E. ps. de P. Pasqual, sig. 590, p. 987 (1795-XII-5).

(82) A. M. E. ps. de P. Pasqual, sig. 589, p. 672 (1793-VIII-9).

(83) A. M. E. ps. de P. Pasqual, sig. 590, p. 931 (1795-VII-13).

(84) A. M. E. ps. de P. Pasqual, sig. 589, p. 722 (1793-XI-22).

(85) A. M. E. ps. de P. Pasqual, sig. 1148, p. 1402 (1799-XI-23).

(86) Pedro RUIZ TORRES: Señores..., op. cit., pp. 340-341.

(87) A. M. E. ps. de P. Pasqual, sig. 590, p. 904 (1795-III-21).

(88) A. M. E. ps. de P. Pasqual, sig. 590, p. 936 (1795-VI-17).

(89) A. M. E. ps. de P. Pasqual, sig. 590, p. 937 (1795-VI-17).

(90) A. M. E. ps. de P. Pasqual, sig. 591, p. 1269 (1798-III-14).

(91) A. M. E. ps. de P. Pasqual, sig. 1148, p. 1369 (1799-IX-30).

(92) A. M. E. ps. de P. Pasqual, sig. 591, p. 1204 (1797-VIII-23).

(93) A. M. E. ps. de P. Pasqual, sig. 1148, p. 1362 (1799-1X-17).

(94) A. M. E. ps. de P. Pasqual, sig. 591, p. 1338 (1798-|X-13).

(95) A. M. E. ps. de P. Pasqual, sig. 1148, p. 1362 (1799-IX-17).

(96) A. M. E. ps. de J. Ardid, sig. 507, p. 179 (1740-1-21).

(97) A. M. E. ps. de J. Ardid, sig. 508, p. 397 (1748-1-1).

(98) A. M. E. ps. de P. Pasqual, sig. 588 , p. 575 (1792-VII-13).

(99) A. M. E. ps. de J. Ardid, sig. 507, p. 7 (1735-IX-30).

(100) A. M. E. ps. de P. Pasqual, sig. 591, p. 1321 (1798-VIIl-1).

(101) A. M. E. ps. de J. Ardid, sig. 508, p. 355 (1746-IV-1).

(102) A. M. E. ps. de P. Pasqual, sig. 587, p. 298 (1790-ill-6).

(103) A. M. E. ps. de P. Pasqual, sig. 586, p. 2 (1786-V-22).

(104) A. M. E. ps. de P. Pasqual, sig. 586, p. 121 (1788-I-16).

(105) A. M. E. ps. de J. Ardid, sig. 508, p. 330 (1745-I-24).

(106) A. M. E. ps. de J. Ardid, sig. 508, p. 402 (1748-III-8).

(107) A. M. E. ps. de J. Ardid, sig. 507, p. 259 (1741-XII-9).

(108) A. M. E. ps. de P. Pasqual, sig. 586, p. 38 (1786-XII-31).

(109) A. M. E. ps. de P. Pasqual, sig. 591, p. 1301 (1798-V-23).

(110) A. M. E. ps. de P. Pasqual, sig. 591, p. 1301 (1798-V-23).

(111) A. M. E. ps. de P. Pasqual, sig. 586, p. 130 (1788-11-26).

(112) A. M. E. ps. de J. Ardid, sig. 510, p. 788 (1766-IV-27).

A. M. E. ps. de J. Ardid, sig. 510, p. 789 (1766-IV-27).

A. M. E. ps. de J. Ardid, sig. 510, p. 790 (1766-IV-27).

(113) P. GOUBERT: Familles marchandes sous l'Ancien Régime: les Danse et les Motte de Beauvais. S. E. V.P. E. N. París, 1959. 192 pp.

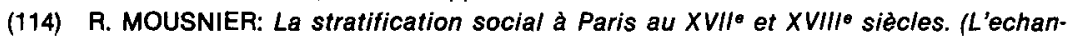
tillon de 1634, 1635, 1636). Pédone. París, 1976.

(115) H. MARTIN: Livre, pouvoirs et société à Paris au XVIle siècle (1598-1701). Droz. Genève, 1969. 2 vols. Analiza 600 inventarios de bibliotecas particulares para conocer la difusión del libro entre lectores de diferentes posiciones socio-económicas.

Y DURANT: "L'histoire sociale nantaise et les archives notariales". En Les Actes Notariés. Actas del Coloquio de Estrasburgo. Lib. Istra, 1979, pp.: 105-121. Observa el contenido de las bibliotecas inventariadas sefhalando qué tipo de libros existen, su temática, quién posee los libros, etc.

(116) K. LOCKRIDGE: "L'alphabetisation en Amérique, 1650-1800». En Annales, 1977, n. 3. 
(117) A. M. E. ps. de P. Pasqual, sig. 586, p. 138 (1788-V-5).

(118) A. M. E. ps. de J. Ardid, sig. 509, p. 616 (1757-V-1).

(119) A. M. E. ps. de P. Pasqual, sig. 586, p. 30 (1786-X-29).

(120) Pedro IBARRA Y RUIZ: Historia de Elche. Manuel Pastor Torres, editor. Colección "Papers d'Elx», 1982, pp. 228-229.

(121) A. M. E. ps. de P. Pasqual, sig. 591, p. 1320 (1798-VII-25). La familia Perpiñán posee una sepultura propia en la iglesia de Santa Maria.

(122) A. M. E. ps. de J. Ardid, sig. 510, p. 822 (1774-III-19).

(123) A. M. E. ps. de P. Pasqual, sig. 586, p. 173 (1788-X-18).

(124) A. M. E. ps. de P. Pasqual, sig. 591, p. 173 (1788-X-18). El testante manda se celebren 600 misas por su alma.

A. M. E. ps. de J. Ardid, sig. 510, p. 822 (1774-III-19). El testante manda se celebren $1010 \mathrm{mi}-$ sas por su alma.

(125) A. M. E. ps. de J. Ardid, sig. 507, p. 211 (1741-1-14).

(126) A. M. E. ps. de J. Ardid, sig. 508, p. 272 (1742-III-28)

(127) A. M. E. ps. de J. Ardid, sig. 507, p. 238 (1741-V-22). El ejemplo más espectacular dentro de este grupo nos lo proporciona el testamento de Marla Antonia Quesada, que manda 10 misas anuales, perpetuas, por su alma, ascendientes y descendientes. A. M. E. ps. de J. Ardid, sig. 507 , p. 44 (1736-V-7).

(128) A. M. E. ps. de P. Pasqual, sig. 1148, p. 1413 (1800-1II-11).

(129) A. M. E. ps. de J. Ardid, sig. 508, p. 360 (1746-VIII-2). Se manda una limosna de dos reales a Nuestra Señora de la Aurora.

(130) A.M.E. ps. de P. Pasqual, sig. 591, p. 1227 (1797-XII-26). El testante lega 20 reales a la Hermandad de la Escuela de Cristo Nuestro Señor de Elche y otros 20 reales a la de Novelda.

(131) A.M.E. ps. de P. Pasqual, sig. 590, p. 1935 (1795-VII-16). La testante reparte cuatro libras entre 30 pobres después de que éstos recen un rosario a la puerta de su casa el día de su entierro.

(132) Ph. ARIES: L'homme devant la mort. Editions du Seuil. Paris, 1977, 642 pp.

(133) Ph. GOUJARD: «Echec d'une sensibilité baroque: les testaments rouennais au XVIIle siècle». En Annales, 1981, número 1, pp. 26-43.

(134) F. LEBRUN: Les hommes et la mort en Anjou aux XVI/ et XVII/ siècles. Mouton. Paris, 1971, 562 pp.

(135) Pierre CHAUNU: La mort á Paris $\left(X V I^{\circ}, X V I^{\circ}\right.$ et $X V I I I^{\circ}$ siècles $)$. Libreria Arthème Fayard, Paris, $1978,543 \mathrm{pp}$.

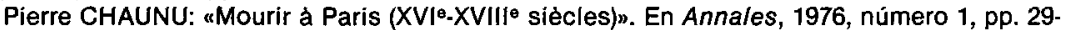
51.

(136) Michel VOVELLE: Piété baroque et déchristianisation en Provence au XVII/ ${ }^{\circ}$ siècle. Editions du Seuil, París, 346 pp. Esta edición es una versión abreviada de la obra publicada bajo el mismo título en la Libreria Plon en 1973 en la colección "Civilisation et mentalités", dirigida por Philippe Ariès et Rober Mandrou.

Michel VOVELLE: "Les attitudes devant la mort: problèmes de méthode, approches et lectures différentes". En Annales, 1976, número 1, pp.: 120-132.

Michel VOVELLE: «Un préalable à toute histoire sérielle: la représentativité sociale du testament (XIV ${ }^{\theta}$ XIX ${ }^{\ominus}$ siècle)". En Les Actes Notariés. Actas del Coloquio de Estrasburgo, Lib. Istra, 1979, pp. 257-277.

(137) Baudilio BARREIRO MALLON: "El sentido religioso del hombre ante la muerte en el Antiguo Régimen. Un estudio sobre archivos parroquiales y testamentos notarialesn. En Actas de las I Jornadas de Metodología Aplicada a las Ciencias Históricas. Vol. V, pp. 181-197. Secretariado de Publicaciones de la Universidad de Santiago de Compostela, 1975. 